\title{
Análisis comparativo de las aves de dos zonas boscosas de Pueblo Viejo, Chiapas, México
}

\section{Comparative analysis of the birds of two forested areas in Pueblo Viejo, Chiapas, Mexico}

\author{
Luis Enrique De los Santos-Vázquez ${ }^{1}$, Marco Antonio Altamirano-González Ortega ${ }^{2 *}$ \\ ${ }^{1}$ WindField S.A. de C.V. Antonio Merlo de La Fuente No. 3, Colonia La Candelaria Teotlalpan. CP. 90168. Totolac, Tlaxcala, \\ México. \\ ${ }^{2}$ Coordinación Técnica de Investigación, Secretaría de Medio Ambiente e Historia Natural. Calzada de los Hombres ilustres s/n. \\ CP. 29000. Tuxtla Gutiérrez, Chiapas, México. \\ *Autor de correspondencia: biomarc2002@yahoo.com.mx
}

Artículo científico recibido: 05 de julio de 2017 aceptado: 13 de abril de 2018

RESUMEN. La falta de información básica sobre poblaciones de aves, ha limitado realizar propuestas de conservación local. Por lo anterior, el objetivo fue comparar la avifauna de dos localidades, ubicadas entre los municipios de Las Rosas y Comitán de Domínguez, en Chiapas, México. El registro de individuos se realizó en 80 puntos de conteo, a las especies registradas se les asignó estatus de temporalidad de residencia y riesgo, de sensibilidad a disturbios provocados por actividades humanas y gremio trófico, además se realizaron análisis de abundancia relativa y de números de Hill, para reconocer especies raras. También se estimó la diversidad beta y complementariedad de especies, entre localidades. Se registraron 113 especies, 86 residentes, 22 migratorias invernales, una migratoria de verano y cuatro transitorias. La más alta riqueza y abundancia de especies se registró en Candelaria en la temporada de secas, siendo la mayoría rara. Candelaria tiene 15 especies en riesgo, 55 tienen baja sensibilidad a disturbios, 44 media y dos alta. En Guadalupe Palmira el registro de especies fue menor en todas las categorías. Las especies fueron principalmente insectívoras (56 especies) y omnívoras arbóreas (19 especies), la diversidad beta fue del 39\% y la complementariedad del $56 \%$.

Palabras clave: Abundancia, diversidad, riesgo, riqueza, sensibilidad

ABSTRACT. The lack of basic information on bird populations has limited the ability to formulate local conservation proposals. Therefore, the objective was to compare the avifauna of two localities, located between the municipalities of Las Rosas and Comitán de Domínguez in Chiapas, Mexico. Individuals were recorded at 80 counting points, with the recorded species being assigned temporary residence and risk status, sensitivity to disturbances caused by human activities and trophic guild, and an analysis of relative abundance and Hill numbers was performed to recognize rare species. Beta diversity and complementarity of species, between locations, were also estimated. A total of 113 species were recorded, consisting of 86 residents, 22 winter migrants, one summer migrant and four transient ones. The highest richness and abundance of species were recorded in Candelaria in the dry season, the majority being rare. Candelaria has 15 species at risk, 55 have low sensitivity to disturbances, 44 medium and two high. In Guadalupe Palmira the recording of species was lower in all categories. The species were mainly insectivores (56 species) and arboreal omnivores (19 species); beta diversity was $39 \%$ and complementarity $56 \%$.

Key words: Abundance, diversity, risk, richness, sensitivity

\section{INTRODUCCIÓN}

La avifauna en México es muy diversa, con un registro entre 1123 y 1150 especies, lo que equivale al $11 \%$ de las aves conocidas de mundo (Navarro-Sigüenza et al. 2014). El estado de Chiapas tiene un elevado número de especies, con una riqueza potencial de hasta 732 especies de aves 


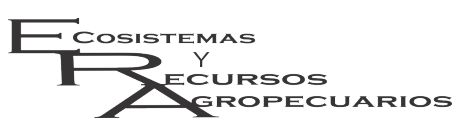

que se distribuyen en muchas de las áreas naturales protegidas que se han decretado en el estado (Rangel-Salazar et al. 2013). A pesar de las 43 áreas naturales protegidas (18 federales y 25 estatales) del estado, estas representan el $18.4 \%$ del territorio estatal y muchas presentan diferente grado de deterioro ambiental, como deforestación, crecimiento agropecuario, crecimiento de la frontera urbana, tala ilegal, cacería, tráfico de flora y fauna, introducción de especies exóticas, invasión de tierras, incendios forestales y contaminación de aguas (Jiménez 2013).

La comunidad de Pueblo Viejo, propuesta como área natural protegida, es un área ubicada entre los municipios de Las Rosas y una pequeña parte de Comitán de Domínguez, en el estado de Chiapas (SEMARNAT-CONANP 2009). La falta de información básica sobre la riqueza y la abundancia de las poblaciones de aves en esta área, como en muchas localidades de Chiapas, ha imposibilitado corroborar su condición actual (Rangel-Salazar et al. 2005). La Comisión Nacional de Áreas Naturales Protegidas pretende mediante la compilación de estudios de investigación en diferentes grupos taxonómicos, junto a los de carácter económicosocial, fortalecer una propuesta de conservación y manejo para Pueblo Viejo (SEMARNAT-CONANP 2009), pero no existen estudios sistemáticos que denoten la importancia del área y las necesidades para su conservación que apoyen la propuesta. Por lo que se requiere: i) generar líneas base en diferentes taxones, que establezcan la relación de la riqueza y la abundancia de las especies que allí ocurren; ii) información sobre aquellas especies de aves en alguna categoría de riesgo y con sensibilidad a disturbios provocados por actividades humanas; y iii) análisis comparativos en estos y otros aspectos de la avifauna entre localidades, en las áreas identificadas para su conservación legal, que puedan ser relevantes en la propuesta para reconocerlas y lograr su establecimiento. Por lo anterior, el objetivo fue comparar la avifauna de las localidades Candelaria y Guadalupe Palmira con base en su composición, riqueza, abundancia y diversidad.
De los Santos-Vázquez y Altamirano-González Ortega Comparativo de aves en bosques Ecosist. Recur. Agropec. 5(15):465-475,2018

\section{MATERIALES Y MÉTODOS}

\section{Área de estudio}

Pueblo Viejo se localiza en el municipio de Las Rosas y abarca una pequeña parte del municipio de Comitán de Domínguez, en el estado de Chiapas, con un área de 8352 ha (Figura 1), se ubica entre los $16^{\circ} 20^{\prime} 50^{\prime \prime}$ y $16^{\circ} 25^{\prime} 00^{\prime \prime}$ LN y entre los $92^{\circ} 20^{\prime}$ $05^{\prime \prime}$ y $92^{\circ} 23^{\prime} 00^{\prime \prime}$ LO. Colinda al sur con el ejido San Luis, al este con los bienes comunales del conjunto Candelaria y al norte con el ejido Guadalupe Palmira. Presenta un clima semicálido húmedo con lluvias en verano (García 1988). La vegetación característica es de bosque tropical caducifolio con transición a bosque de coníferas y bosque mesófilo (Rzedowski 2006).

\section{Trabajo de campo}

Se realizaron muestreos mensuales, entre octubre de 2012 y septiembre de 2013, en dos localidades de Pueblo Viejo (Candelaria y Guadalupe Palmira), con duración de cuatro días cada uno, durante 12 meses. En cada localidad, se establecieron cuatro senderos de observación con una extensión de $2.5 \mathrm{~km}$ cada uno (Figura 1), con 10 puntos de conteo cada uno y $50 \mathrm{~m}$ de radio, separados entre sí por $250 \mathrm{~m}$ de distancia (Ralph et al. 1996). El conteo de individuos de las diferentes especies de aves, se realizó por periodos de $10 \mathrm{~min}$, mediante el uso de binoculares $8 \times 42$ y de forma auditiva. El conteo inició al amanecer, aproximadamente a las 6:00 h, concluyendo a las 11:00 h. La determinación de las aves se realizó de forma directa con las guías de campo de Howell y Webb (1995) y Peterson y Chalif (2008). También se tomaron fotografías de algunos individuos de determinación confusa, con una cámara réflex Canon EOS Revel T3 ${ }^{\circledR}$, para posteriormente corroborarlos con las guías de campo o con ejemplares depositados en la Colección Zoológica Regional de Aves de la Secretaría del Medio Ambiente e Historia Natural del Estado de Chiapas. 


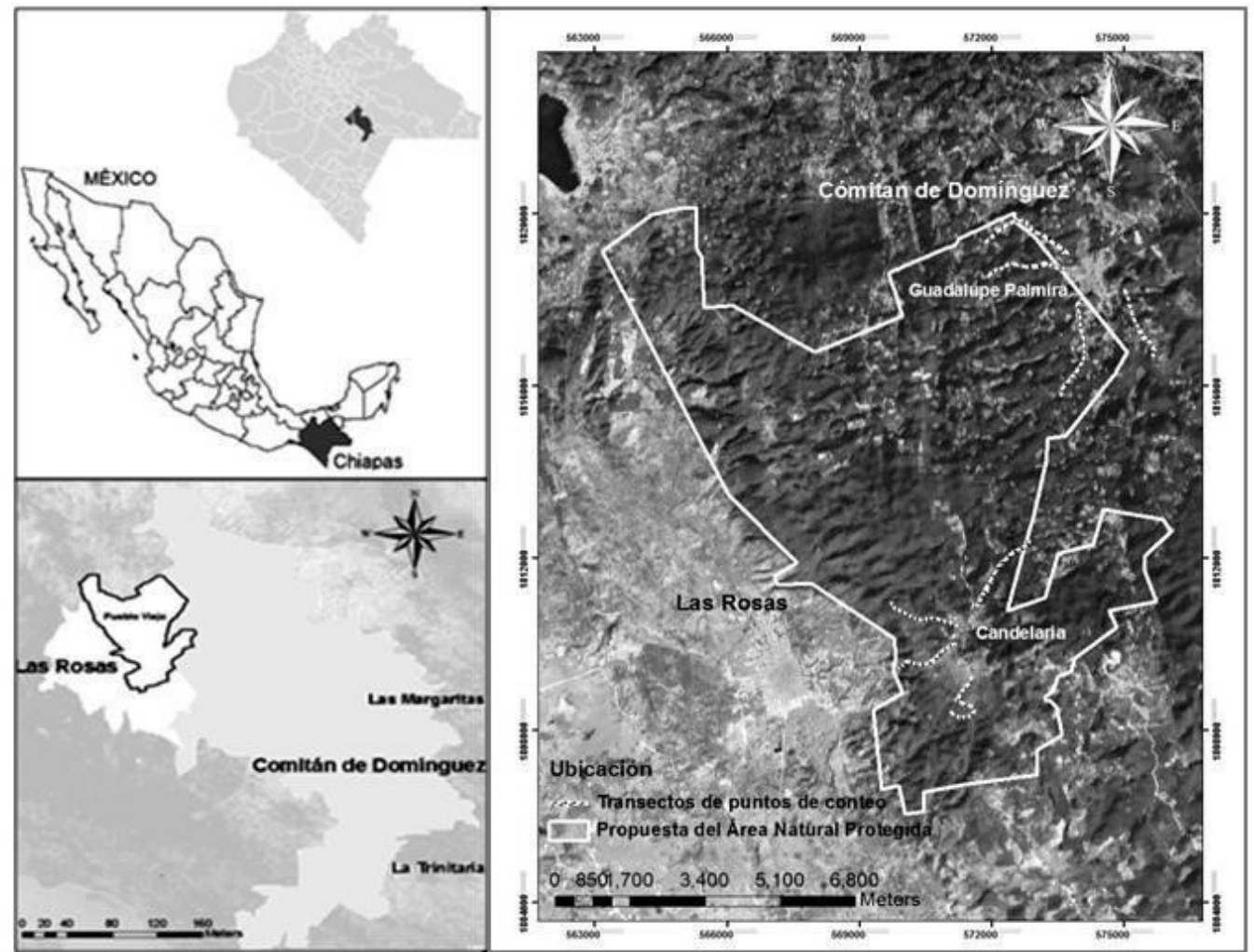

Figura 1. Ubicación del área de estudio y de los senderos de observación donde se localizan las localidades de muestreo.

\section{Asignación de categorías y estimación de parámetros}

Las especies de aves registradas se ubicaron en las categorías taxonómicas de orden, familia, género y especie, consideradas por la American Ornithologists' Union (Chesser et al. 2017). También se asignó a cada especie su nombre común en español e inglés, así como la temporalidad de residencia principal (residente, migratorio de invierno, migratorio de verano y transitorias) propuesta por Howell y Webb (1995) y Berlanga et al. (2015), identificandose aquellos individuos incluidos en alguna categoría de riesgo considerada en la NOM-059-SEMARNAT-2010 (DOF 2015) y la IUCN 2017. La sensibilidad a disturbios provocados por actividades humanas ( $\mathrm{A}=$ alta, $\mathrm{M}=$ media, $\mathrm{B}=$ baja) se asignó con lo propuesto por Stotz et al. (1996), que se basa en la posibilidad que tienen las especies de aves para adaptarse a los ambientes alterados o depender de bosques en buen estado de conservación, además d el gremio trófico al que pertenecen (Rangel-Salazar et al. 2009), para ubicarlas como $\mathrm{RD}=$ rapaz diurna, $\mathrm{GT}=$ granívoro terrestre, $\mathrm{FT}=$ frugívoro terrestre, $\mathrm{FA}=$ frugívoro arbóreo, $\mathrm{Ne}=$ nectarívoro, $\mathrm{OA}=$ omnívoro arbóreo, $\mathrm{IAB}=$ insectívoro arbóreo buscador, IBC = insectívoro buscador de corteza, IBT = insectívoro buscador terrestre e IAR = insectívoro arbóreo recogedor.

La riqueza de especies de las aves se determinó por el número de especies registradas en cada localidad muestreada. La abundancia absoluta de las especies de aves se determinó mediante el número de individuos registrados de cada especie, en cada localidad muestreada. La abundancia relativa se estimó con base en la proporción de individuos de cada especie con relación al total de individuos de las especies en su conjunto (Moreno 2005). Con los 


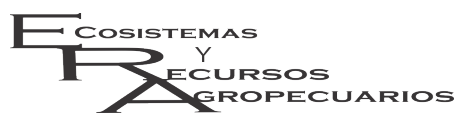

datos de riqueza y abundancia obtenidos por localidad, se realizó un análisis mensual de la información y su interpretación por temporada de secas, que va de octubre a abril y la de lluvias, de mayo a septiembre.

Se construyeron curvas de especieabundancia para cada localidad y el análisis de números efectivos de especies (qD) conocido como números de Hill (Jost 2006), bajo dos aproximaciones: 1) dándole mayor peso a las abundancias de las especies raras, $q=0.5$; y 2 ) otorgando un mayor peso a las especies abundantes, $q=2$ (Moreno et al. 2011, Muñoz-Alonso et al. 2017). La diversidad beta de las especies de aves, se estimó utilizando la modificación propuesta por Halffter y Moreno (2005), con la fórmula (diversidad total / diversidad promedio) - $1 * 100$, y mediante un análisis de complementariedad de especies (Colwell y Coddington 1994), se obtuvo el porcentaje de disimilitud (diferencia) en la composición de especies entre localidades. La expresión utilizada fue $\mathrm{CAB}=(\mathrm{UAB}$ ( $\mathrm{SAB}) * 100$, donde UAB es el número de especies de aves únicas a cualquiera de las dos localidades $(a+b-2 c)$, SAB es la riqueza total de aves para ambas localidades combinadas $(a+b-c)$, a es el número de especies de aves de la localidad $A, b$ es el número de especies de aves de la localidad $\mathrm{B}, \mathrm{y}$ $c$ es el número de especies de aves en común entre las localidades A y B.

\section{RESULTADOS}

Se contabilizaron 2747 individuos en las dos localidades que pertenecen a 113 especies, 11 órdenes y 34 familias. El orden de los Passeriformes presentó el mayor número de familias (21). Las familias que tuvieron el mayor número de especies entre las dos localidades muestreadas fueron Parulidae (17 spp.), Icteridae (9 spp.), Turdidae (8 spp.), Tyrannidae (7 spp.) y Emberizidae (6 spp.). Por otra parte, 86 especies $(76 \%)$ son residentes, 22 migratorias invernales (19\%), una migratoria de verano $(1 \%)$ y cuatro transitorias ( $4 \%)$.

Los meses con mayor riqueza de especies fue-
De los Santos-Vázquez y Altamirano-González Ortega Comparativo de aves en bosques Ecosist. Recur. Agropec. 5(15):465-475,2018

ron febrero y abril de 2013 con 53 especies (47\%), seguido de diciembre de 2012 con 51 (45\%), junio de 2013 con 44 (39\%), y octubre de 2012 con 41 (36\%) que corresponden con la temporada de secas, con excepción del mes de junio. Los meses con mayor abundancia fueron diciembre de 2012 con 350 individuos (13\%), febrero de 2013 con 336 (12\%) y abril de 2013 con 333 (12\%), que corresponden a la temporada de secas.

Las especies más abundantes presentaron entre 96 y 264 individuos, Campylorhynchus zonatus con 96 individuos, Cyanocitta stelleri con 120, Trogon mexicanus con 106, Icterus chrysater con 99, Turdus rufitorques con 264, Ptiliogonys cinereus con 222 y Zonotrichia capensis con 99. Las abundancias relativas mayores ( $\geq 6$ individuos), correspondieron a cinco especies de aves, con una especie común en las dos localidades muestreadas (Turdus rufitorques). La mayoría de las especies en las dos localidades muestreadas corresponden a la categoría rara (49 especies).

De las 113 especies, 15 están incluidas en alguna categoría de riesgo, consideradas en la NOM059-SEMARNAT-2010, dos están en peligro de extinción, ocho amenazadas y cinco están sujetas a protección especial. Mientras que Setophaga chrysoparia y Penelopina nigra se encuentran bajo las categorías de peligro de extinción y vulnerable, de manera respectiva en la Lista Roja de especies en riesgo de la IUCN, el resto de las especies se consideran bajo la categoría de preocupación menor. La mayoría de las aves corresponden a la clasificación de sensibilidad baja (B) de disturbios provocados por actividades humanas (62 especies); 48 están en la categoría media (M) y tres en la categoría alta (A). Con relación a las categorías de gremios tróficos, cinco especies se ubican en la categoría rapaz diurna (RD), 12 en granívoro terrestre (GT), ocho en frugívoro terrestre (FT), 10 en frugívoro arbóreo (FA), tres en nectarívoro $(\mathrm{Ne})$, 19 en omnívoro arbóreo (OA), 12 en insectívoro arbóreo buscador (IAB), 10 en insectívoro buscador de corteza (IBC), dos en insectívoro buscador terrestre (IBT) y 32 en insectívoro arbóreo recogedor (IAR). 


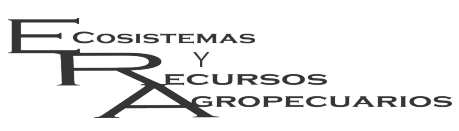

De los Santos-Vázquez y Altamirano-González Ortega Comparativo de aves en bosques Ecosist. Recur. Agropec. 5(15):465-475,2018

\section{Comparativo entre localidades}

En Candelaria, se contabilizaron 1578 individuos, distribuidos en 101 especies, 32 familias y 11 órdenes, mientras que en Guadalupe Palmira el registro de individuos fue de 1169 distribuidos en 62 especies, 24 familias y seis órdenes. Los órdenes que presentaron mayor número de familias en Candelaria fueron los Passeriformes con 19 familias, Parulidae con 17, Icteridae con nueve y Turdidae con ocho. En Guadalupe Palmira, los órdenes con mayor número de familias también fueron los Passeriformes con 19 familias: Parulidae con 14 y Fringillidae con cinco (Figura 2). Con relación a la estacionalidad, 76 especies fueron residentes, 20 migratorias invernales, una migratoria de verano y cuatro transitorias en Candelaria, mientras que en Guadalupe Palmira, 46 especies son residentes, 14 migratorias invernales y dos transitorias (Figura 3). En cuanto a especies exclusivas, 51 ocurren en Candelaria y 12 en Guadalupe Palmira.

La mayor riqueza de especies en Candelaria se observó en febrero y abril de 2013 con 53 especies $(47 \%)$, en la temporada de secas, mientras que en Guadalupe Palmira se presentó en los meses de mayo de 2013, con 40 especies (35\%) y noviembre de 2012 con 38 (34\%), en la temporada de lluvias y secas, respectivamente (Figura 4). El mayor número de individuos en Candelaria, ocurrió en diciembre de 2012 con 350 (13\%), mientras que en Guadalupe Palmira fue en marzo de 2013, con 250 (9\%), los dos meses en temporada de secas. El análisis de abundancia reveló la presencia de especies en su mayoría raras (Figura 5). En Candelaria (a), fueron 65 especies para $\mathrm{q}<1$ (raras) y 36 especies para $\mathrm{q}>1$ (abundantes), mientras que en Guadalupe Palmira (b), fueron 36 especies para $\mathrm{q}<1$ (raras) y 26 especies para $\mathrm{q}>1$ (abundantes).

Con base en la NOM-059-SEMARNAT-2010, en Candelaria hay 13 especies de aves incluidas en alguna categoría de riesgo: dos en peligro de extinción, seis amenazadas y cinco sujetas a protección especial. De acuerdo con la Lista Roja de especies en riesgo una está en peligro de extinción, una vulnerable y 99 en preocupación menor. En Guadalupe Palmira el registro de especies de aves incluidas en la normatividad nacional es de cinco: una en peligro de extinción y cuatro amenazadas. Por la IUCN, una se encuentra en peligro de extinción y en preocupación menor 61 especies. Las especies de aves con sensibilidad a disturbios provocados por actividades humanas, registradas en Candelaria son 55 especies en la categoría baja (B), 44 en media (M) y dos en alta (A). Mientras que en Guadalupe Palmira se registraron 34 especies en la categoría baja (B), 26 en media (M) y en alta (A) dos especies (Tabla 1). La distribución de las aves en los gremios tróficos para Candelaria, fue de 10 especies como frugívoro arbóreo (FA), seis frugívoro terrestre (FT), ocho granívoro terrestre (GT), 32 insectívoro arbóreo recogedor (IAR), 12 insectívoro arbóreo buscador (IAB), siete insectívoro buscador de corteza (IBC), dos insectívoro buscador terrestre (IBT), dos nectarívoro (Ne), 17 omnívoro arbóreo (OA) y cinco rapaz diurna (RD). En Guadalupe Palmira dos especies de aves son FA, tres FT, seis GT, 20 IAR, cuatro IAB, cuatro IBC, dos $\mathrm{Ne}$, ocho $\mathrm{OA}$ y una RD. La diversidad beta estimada para las especies de aves entre Candelaria y Guadalupe Palmira fue del $39 \%$, mientras que el valor de complementariedad señaló una diferencia entre estas localidades del $56 \%$.

\section{DISCUSIÓN}

Las diferencias en la composición de las especies de aves entre Candelaria y Guadalupe Palmira, son contrastantes, lo que puede deberse a los diferentes tipos de bosques que existen en cada una de ellas y por las diferencias en la heterogeneidad ambiental. En Candelaria hay más tipos de vegetación boscosa que en Guadalupe Palmira y se observa una mayor heterogeneidad ambiental. El registro de las especies migratorias en Candelaria tuvo el mayor porcentaje (22\%) de la totalidad de aves registradas en Pueblo Viejo. Este fenómeno, ocurrió con un mayor registro de individuos en los meses de diciembre, febrero y abril que corresponden con la temporada de migración registrada para la mayoría de las aves en el Neotrópico (USFWS 2005). Al respecto se señala que la heterogenei- 


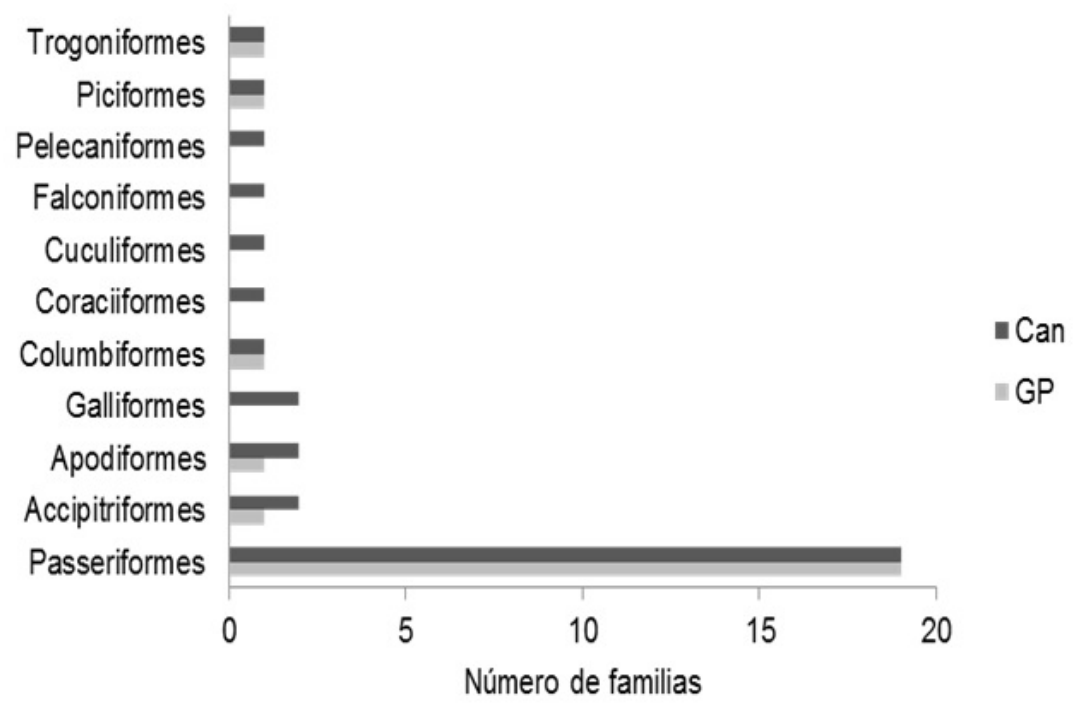

Figura 2. Número de familias por orden taxonómico de la comunidad de aves registradas en Candelaria (Can) y Guadalupe Palmira (GP).

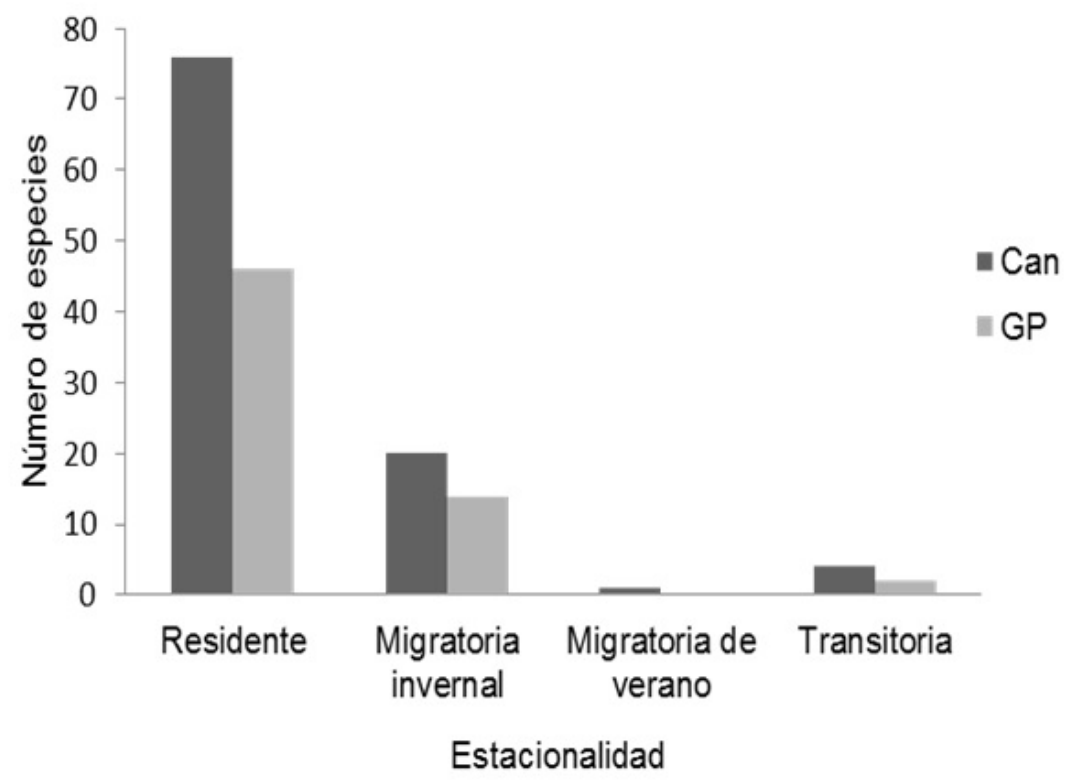

Figura 3. Estacionalidad de las especies de aves, registradas en Candelaria (Can) y Guadalupe Palmira (GP).

dad ambiental afecta la dispersión de las especies y la selección del hábitat (Freemak y Merriam 1986). No obstante que la proporción de aves que arriban, contribuye de manera considerable al aumento en el número de especies e individuos totales para la avifauna de Pueblo Viejo, donde la presencia de aves residentes es mayor. Al respecto Kennedy et al. (2010), indica que este fenómeno depende de la heterogeneidad del paisaje, provocada por la fragmentación del hábitat.

A pesar de que en Pueblo Viejo se registró un bajo porcentaje de especies de aves de Chiapas 

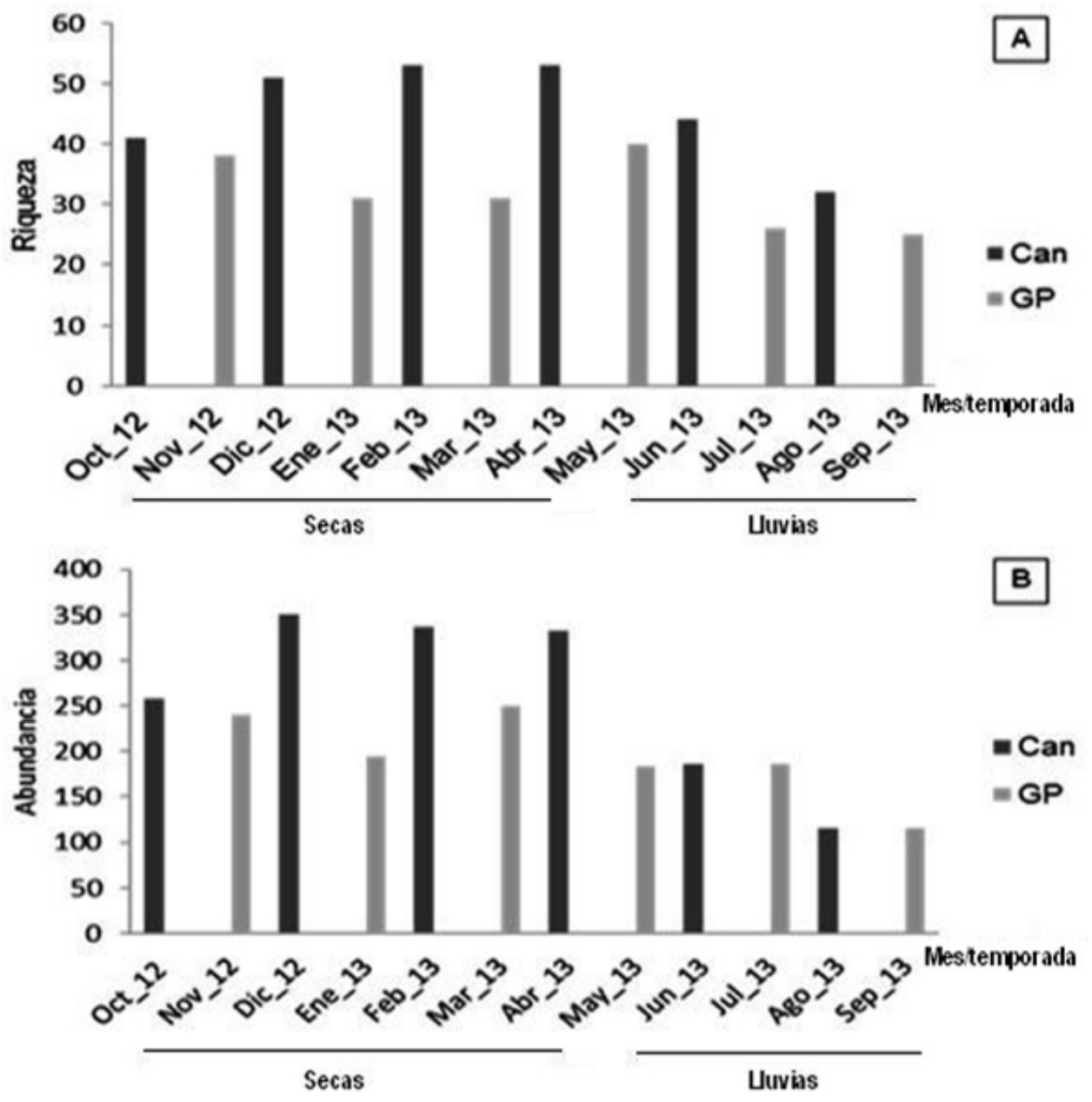

Figura 4. Riqueza (A) y abundancia (B) de las especies de las aves registradas en Candelaria (Can) y Guadalupe Palmira (GP).

listadas en la NOM-059-SEMARNAT-2010 (DOF 2015 ), con $8.5 \%$ y en la IUCN (IUCN 2017) con $6 \%$, se registraron especies como $S$. chrysoparia que se encuentra en peligro en ambas clasificaciones y $P$. nigra que se registró exclusivamente en Candelaria, donde su presencia puede ser importante, ya que es una especie considerada en peligro de extinción y vulnerable, además de ser una especie reguladora dentro de la cadena trófica (González-García 2000) debido a que se alimenta de frutos, hojas verdes, diferentes tipos de insectos y algunos pequeños vertebrados, como ratones y salamandras. En Guadalupe Palmira se registró S. chrysoparia, que dentro del área muestreada representa uno de los primeros sitios en Chiapas de internación durante su migración hacia Nicaragua (Macías-Caballero 2013). El registro de un elevado número de especies residentes, junto con la presencia de especies migratorias en riesgo, pueden ser aspectos que se consideren para apoyar la propuesta de conservación del área de estudio como área natural protegida. Las especies $S$. chrysoparia y $P$. nigra, están consideradas en la categoría más alta de riesgo establecida por la normatividad de especies en riesgo nacional (DOF 2015) e internacional (IUCN 2017), con un alto grado de vulnerabilidad a los cambios ambientales (Stotz et al. 1996).

Un aspecto relevante fue el registro de es- 

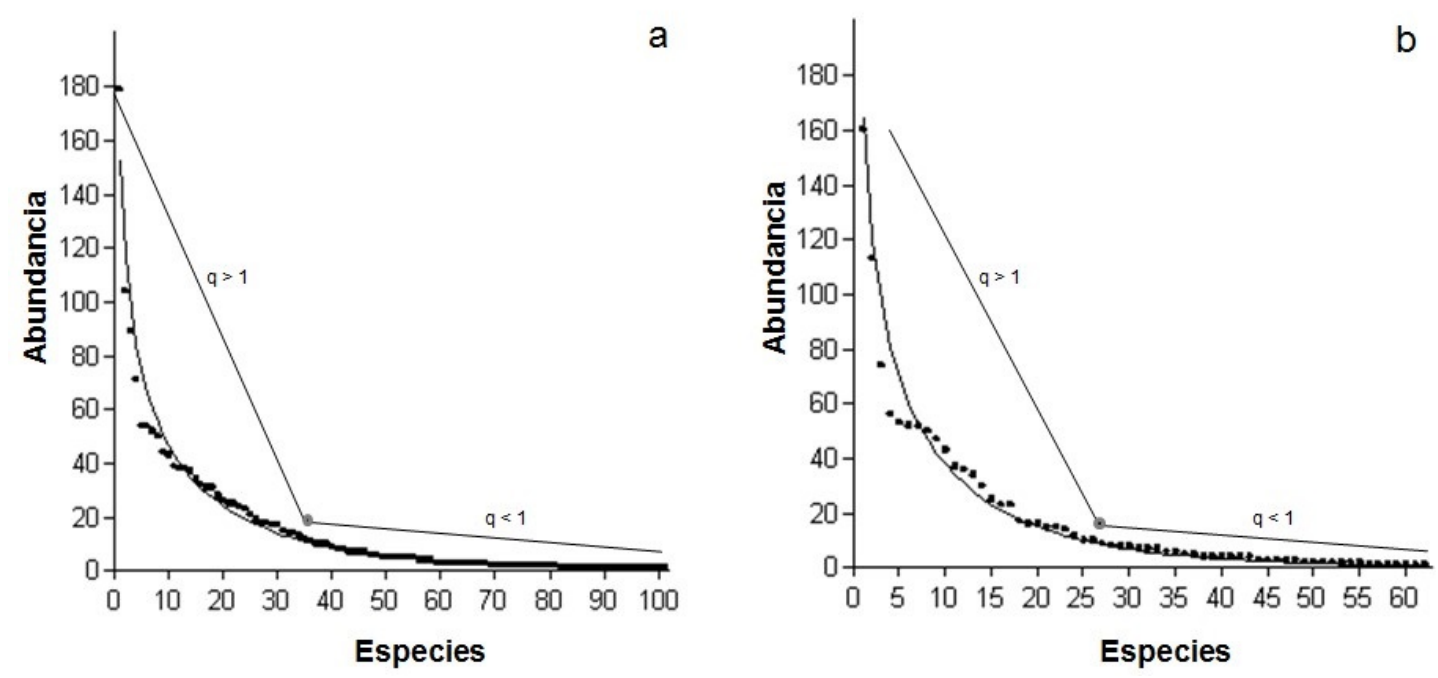

Figura 5. Abundancia de las especies de aves registradas en Candelaria (a) y Guadalupe Palmira (b). $\mathrm{q}<1=$ especies raras; q $>1=$ especies abundantes.

Tabla 1. Especies de aves en Pueblo Viejo y por área muestreada, en categoría de riesgo y de sensibilidad a disturbios provocados por actividades humanas.

\begin{tabular}{cccccc}
\hline Área & $\begin{array}{c}\text { NOM-059-SEMARNAT 2010 } \\
\text { (DOF 2015) }\end{array}$ & IUCN & $\begin{array}{c}\text { Sensibilidad } \\
\text { Alta }\end{array}$ & $\begin{array}{c}\text { Sensibilidad Media } \\
\text { (Stotz et al. 1996) }\end{array}$ & $\begin{array}{c}\text { Sensibilidad } \\
\text { Baja }\end{array}$ \\
\hline Pueblo Viejo & 15 & 2017 & 3 & 48 & 62 \\
Candelaria & 13 & 2 & 2 & 44 & 55 \\
Guadalupe Palmira & 5 & 1 & 2 & 26 & 34 \\
\hline
\end{tabular}

pecies de aves sensibles a los cambios ambientales, ya que un gran porcentaje se ubican en la categoría entre media y baja, tanto para el área total como en cada localidad muestreada. Al respecto, Bonier et al. (2007) predicen que las especies con una amplia tolerancia ambiental, como las 48 especies registradas en la categoría media, podrían adaptarse mejor a los cambios en el hábitat que aquellas especies con poca tolerancia. En este sentido, las especies registradas se distribuyeron en un $66 \%$ dentro de los gremios tróficos insectívoro (56 especies) y omnívoro arbóreo (19 especies), que es donde de manera regular se ubican las especies de aves con tolerancia a los cambios ambientales (Stotz et al. 1996). El comparativo de especies realizado entre las localidades muestreadas indica que la mayor riqueza y abundancia de aves se encuentra en Candelaria, principalmente en la temporada de secas. Al respecto Poulin et al. (1992) indican que la mayoría de las especies de aves en la región Neotropical se reproducen antes de la estación de lluvias, posiblemente como respuesta al aumento en la abundancia de artrópodos.

La diversidad beta indica que las localidades estudiadas están ligadas por una pequeña dispersión de especies de aves, que a pesar de ser baja puede contribuir con el mantenimiento de la diversidad de la región. En este sentido y asociando el valor de complementariedad que es de más del $50 \%$, se puede inferir que la transición de Bosque Tropical Caducifolio-Coníferas a Bosque Mesófilo, puede estar beneficiando el movimiento de algunas especies de aves; así como la corta distancia que hay entre localidades, como lo señalan Freemak y Merriam (1986). Candelaria presenta una vegetación compuesta por remanentes de estos tres tipos de vegetación, lo que puede estar propiciando una mayor disponibilidad de hábitats que las aves utilizan (Cody 1974). 


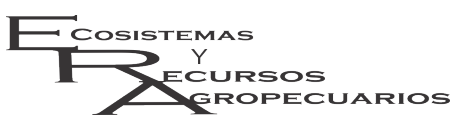

\section{CONCLUSIONES}

Se observaron 113 especies de aves en Pueblo Viejo y se evidencia la importancia de la riqueza y abundancia en las localidades muestreadas. La información obtenida sobre especies de aves en alguna categoría de riesgo, temporalidad de residencia, sensibilidad a disturbios provocados por actividades humanas, gremios tróficos y rareza, puede contribuir en las estrategias de conservación y manejo. También puede apoyar la propuesta de creación del área natural protegida Pueblo Viejo y contribuir en su manejo.

\section{AGRADECIMIENTOS}

A David Alberto Muñoz Zetina, Adán Enrique Gómez González y Miguel Ángel Peralta Meixuerio, por sus contribuciones para mejorar la investigación. A Luis Arturo Álvarez Márquez por su acompañamiento en los muestreos realizados. A los pobladores de Candelaria y Guadalupe Palmira por el apoyo para realizar el trabajo de campo. A Elizabeth Coutiño Alfaro por apoyar en la traducción del resumen, al inglés.

\section{LITERATURA CITADA}

Berlanga H, Gómez de Silva H, Vargas-Canales VM, Rodríguez-Contreras V, Sánchez-González LA, OrtegaÁlvarez R, et al. (2015) Aves de México: Lista actualizada de especies y nombres comunes. CONABIO. México. 117p.

Bonier F, Martin P, Wingfield J (2007) Urban birds have broader environmental tolerance. Biological Letters 3: $670-673$.

Brower JE, Zar JH, Von Ende CN (1997) Field and laboratory methods for general ecology. McGraw-Hill. Massachussetts, USA. 273p.

Chesser RT, Burns KJ, Cicero C, Dunn JL, Kratter AW, Lovettte IJ, et al. (2017) Fifty-eighth supplement to the American Ornithologists' Union Check-list of North American Birds. The Auk: Ornithological Advances 134: 751-773.

Cody ML (1974) Competition and the structure of bird communities. Princeton University Press. New Jersey. USA. 326p.

Colwell RK, Coddington JA (1994) Estimating terrestrial biodiversity through extrapolation. Philosophical Transactions of the Royal Society of London 345: 101-118.

DOF (2015) Proyecto de modificación del Anexo Normativo III. Lista de especies en riesgo de la Norma oficial Mexicana NOM-059-SEMARNAT-2010, Protección ambiental-Especies nativas de México de flora y fauna silvestres-Categorías de riesgo y especificaciones para su inclusión, exclusión o cambioLista de especies en riesgo, publicada el 30 de Diciembre de 2010. Diario Oficial de la Federación. 18 de Noviembre de 2015.

Freemak KE, Merriam HG (1986) Importance of area and habitat heterogeneity to bird assemblages in temperate forest fragments. Biological Conservation 36: 115-141.

García E (1988) Modificaciones al sistema de Clasificación Climática de Köppen. $5^{a}$ edición. Talleres Offset Larios SA, México. 120p.

González-García F (2000) Pajuil (Penelopina nigra). En: Ceballos G, Márquez VL (coord.) Las aves de México en peligro de extinción. UNAM. México. pp: 137-139. 
Halffter G, Moreno CE (2005) Significado biológico de las diversidades alfa, beta y gamma. En: Halffter G, Soberón J, Koleff P, Melic A (ed) Sobre diversidad biológica, el significado de las diversidades alfa, beta y gamma. Monografías Tercer Milenio. Zaragoza, España. pp: 5-18.

Howell S, Webb S (1995) A guide to the birds of México and northern Central America. Oxford University Press. Oxford, UK. 851p.

IUCN (2017) The IUCN Red List of Threatened Species. Version 2017-3. http// www.iucnredlist.org. Fecha de consulta 13: de febrero de 2018.

Jiménez GFJ (2013) Las áreas naturales protegidas federales. En: González-Espinoza M, Ramírez- Marcial N, Ruiz-Montoya L (coords.) Diversidad Biológica en Chiapas. PYV, ECOSUR y COCyTECH. México. pp: 391-396.

Jost L (2006) Entropy and diversity. Oikos 113: 363-375.

Kennedy CM, Marra PP, Fagan WF, Neel MC (2010) Landscape matrix and species traits mediate responses of Neotropical resident birds to forest fragmentation in Jamaica. Ecological Monographs 80: 651-669.

Ludwing, JA, Reynolds JF (1988) Statistical Ecology: a primer on methods and computing. Jhon Wiley y Sons. New York, USA. 337p.

Macías-Caballero C (2013) Conservación a escala regional a través de una especie bandera. En: CONABIO (Comp.) La biodiversidad en Chiapas: Estudio de Estado. Comisión Nacional para el Conocimiento y Uso de la Biodiversidad, Gobierno del Estado de Chiapas, México. pp: 338-341

Moreno CE (2005) Métodos para medir la biodiversidad. MyT- Manuales y Tesis. SEA. Vol. 1. Zaragosa. $84 \mathrm{p}$.

Moreno CE, Barragán F, Pineda NP (2011) Reanálisis de la diversidad alfa: alternativas para interpretar y comparar información sobre comunidades ecológicas. Revista Mexicana de Biodiversidad 82: 1249-1261.

Muñoz-Alonso LA, Nieblas-Camacho J, Chau-Cortez MA, González-Navarro AB, López-Pérez J, Pérez-López J (2017) Vulnerabilidad social y biológica ante el cambio climático en la Reserva de la Biosfera Selva El Ocote. En: Ruiz-Montoya L, Álvarez-Gordillo G, Ramírez-Marcial N, Cruz-Salazar B (ed.). Vulnerabilidad social y biológica ante el cambio climático en la Reserva de la Biosfera Selva El Ocote. México. El Colegio de la Frontera Sur, San Cristóbal de Las Casas, Chiapas, México. pp: 395-448.

Navarro-Singüenza AG, Rebón-Gallardo MF, Gordillo-Martínez A, Townsend-Peterson A, Berlanga-García H, Sánchez-González LA (2014) Biodiversidad de aves de México. Revista Mexicana de Biodiversidad 85: S476-S495.

Peterson RT, Chalif EL (2008) Aves de México. Guía de campo. Editorial Diana. Primera Edición. México. 459p.

Poulin B, Lefebvre G, McNeil R (1992) Tropical avian fenology in relation to abundance and explotation of food resources. Ecology 73: 2295-2309.

Ralph CJ, Geupel GR, Pyle P, Martin TE, Desantafe DF, Milá B (1996) Manual de métodos de campo para el monitoreo de aves terrestres. Gen. Tech. Rep. PSW- GTR-159. United States, Department of Agriculture. Forest Service, Pacific Southwest Research Station. Albany, California. 46p.

Rangel-Salazar, JL, Enríquez PL, Will T (2005) Diversidad de aves de Chiapas: Prioridades de investigación para su conservación. En: González-Espinoza M, Ramírez-Marcial N, Ruiz-Montoya L (Coords.) Diversidad Biológica en Chiapas. PYV, ECOSUR y COCyTECH. México. pp: 265-323. 
Rangel-Salazar, JL, Enríquez PL, Sántiz-López EC (2009) Variación de la diversidad de aves de sotobosque en el Parque Nacional Lagos de Montebello, Chiapas, México. Acta Zoológica Mexicana 25: 479-495.

Rangel-Salazar JL, Enríquez-Rocha PL, Altamirano González-Ortega MA, Macías Caballero C, Castillejos Castellanos E, González Domínguez P, et al. (2013) Diversidad de aves: Un análisis espacial. En: CONABIO (comp.) La biodiversidad en Chiapas: Estudio de estado. Comisión Nacional para el Conocimiento y Uso de la Biodiversidad, Gobierno del Estado de Chiapas. pp: 329-337.

Rzedowski J (2006) Vegetación de México. 1ra. Edición digital. Comisión Nacional para el Conocimiento y Uso de la Biodiversidad. México. 504p.

SEMARNAT-CONANP (2009) Estudio previo justificativo para establecer el área de protección de los recursos naturales Pueblo Viejo, municipio Las Rosas, Chiapas, México. SEMARNAT. Chiapas, México. 30p.

Shannon CE (1948) The mathematical theory of communication. The Bell System Technical Journal 27: 379-423.

Stotz, DF, Fitzpatrick JW, Parker TA, Moskovits DK (1996) Neotropical birds: ecology and conservation. University of Chicago Press. Illinois, USA. 478p.

USFWS (2005) Neotropical Migratory Birds Conservation Act. North American Migratory Birds List. http://www.fws.gov/birdhabitat/Grants/NMBCA/Bird List.shtm. Fecha de consulta: 15 de julio de 2016. 
